\title{
Management of Patients with Oral Cancer during the Covid-19 Pandemic
}

\author{
Solano Nicolás ${ }^{1,2}$, Gutierrez Paulina ${ }^{1}$, Parra Enmanuel ${ }^{1,3^{*}}$ and Castrillo Ariamay ${ }^{3}$ \\ ${ }^{1}$ Oral and Maxillofacial Surgeon, Oral and Maxillofacial Surgery Unit, University Hospital of Maracaibo, Venezuela \\ ${ }^{2}$ Professor, Oral Surgery Post-Graduated Program, School of Dentistry, Universidad del Zulia, Venezuela \\ ${ }^{3}$ D.D.S. Resident, Oral Surgery Post-Graduated Program, School of Dentistry, Universidad del Zulia, Venezuela
}

\begin{abstract}
The influence of the COVID-19 pandemic on healthcare to the public is severe. Healthcare workers are in close contact with infected patients. Transmission of the virus seems to occur mainly by respiratory droplets. There is a high viral load in the nasal and oral cavities of infected patients, especially endangering those specialties focused around this region. Within the field of action of oral and maxillofacial surgeons, the approach and management of oral cancer is one relevant area. Its early approach plays a key role in reducing morbidity and mortality. The present narrative-style review aims to collect and discuss aspects of the management of inpatients and outpatients with oral cancer during the COVID-19 pandemic. It is imperative to follow strict biosecurity protocols when caring for patients with oral cancer during the COVID-19 pandemic.
\end{abstract}

\section{Keywords}

COVID-19, SARS-CoV-2, Oral cancer and oral cancer management

\section{Introduction}

In late December 2019, a cluster of cases of SARS-like viral pneumonia emerged in Wuhan, Hubei province, China. In the following months, the disease spread rapidly to the whole of China as well as more than 120 countries worldwide, and the number of patients infected has been gradually increasing [1]. Corona virus disease (COVID-19) is caused by the SARSCov 2 virus. The World Health Organization gave this disease the name of COVID-19 on February $11^{\text {th }}, 2020$. Outbreaks due to corona virus had occurred before, such as the Severe Acute Respiratory Syndrome (SARS) [2]. Healthcare workers are in close contact with infected patients. Transmission of the virus seems to occur mainly by respiratory droplets [3]. There is a high viral load in the nasal and oral cavity of infected patients, especially endangering those specialties focused around this region. Besides ear, nose, and throat surgeons and dentists, oral and maxillofacial surgeons especially have to be aware of the new challenges and the risk of virus transmission between patients and medical staff. Within the field of action of oral and maxillofacial surgeons, the approach and management of oral cancer is one relevant area [4]. Squamous cell carcinoma represents the most common form of head and neck cancer, and its early approach plays a key role in reducing morbidity and mortality [5]. Based on this, the present narrative-style review aims to collect and to discuss aspects of approaching the management of inpatients and outpatients with oral cancer during the COVID-19 pandemic.

\section{Methods}

The following electronic bibliographic databases were used to identify relevant scientific literature: Science Direct, Google search engine, MEDLINE, SCIELO EMBASE, and CINAHL. A literature search was conducted for English-language articles and free-text keywords to identify eligible reports. The search items used were "corona virus disease 19, COVID-19, SARS-CoV-2, transmission, pandemic, oral surgical procedures, oral and maxillofacial oncology surgery, infection prevention and control, oral cancer." The last search was run on April 29th 2020 . The objective was to identify control and infection prevention strategies during the management of inpatients and outpatients with oral cancer on the COVID-19 pandemic.

* Corresponding author: Enmanuel David Parra, Department of Oral and Maxillofacial Surgery, Dentistry Service, University Hospital of Maracaibo, 16th Avenue, Zulia, Venezuela, Tel: $+58424203607$

Received: May 01, 2020

Accepted: July 02, 2020

Published online: July 04, 2020

Citation: Nicolás S, Paulina G, Enmanuel P, et al. (2020) Management of Patients with Oral Cancer during the Covid-19 Pandemic. Sch J Emerg Med Crit Care 4(1):75-78 


\section{Observations}

\section{Transmission of oral and maxillofacial surgeons in their practice}

Angiotensin II converting enzyme (ACE2) is the cellular receptor through which the virus enters cells. The expression of ACE2 in the oral cavity has been observed especially in the tongue, and thus it is as a potentially high risk site for infectious susceptibility of the virus. ACE2 receptors have also been observed on lymphocytes within the oral mucosa; similar results were found in various organs of the digestive system and the lungs. Although SARS-CoV-2 infection shows no oral signs or symptoms, ACE2 expression in the oral mucosa detects that the orofecal infection route cannot be excluded. Due to this fact and the field of action of surgeons, standard protective measures in daily clinical practice are not effective enough to prevent transmission of the virus. Oral and maxillofacial surgeons are directly exposed to inhalation of viral particles in aerosols (where the virus can remain viable for up to 3 hours), especially when patients are in the incubation period, unaware that they are infected, putting the attention staff at high risk. Therefore, it is imperative to refine preventive strategies, reducing the activity of the surgeon to strict emergencies to cut the chain of contagion [6].

The use of personal protective equipment should be selected adequately and used appropriately. Currently, there are no specific guidelines for the protection of healthcare workers such as oral and maxillofacial surgeons dealing with oncology procedures in the head and neck area, specifically in the oral cavity. Because of the frequent exposure to saliva, sputum, and other body fluids, surgeons are exposed to a high risk of viral transmission [4]. When performing a clinical examination of the patient with oral cancer wearing a surgical mask, the use of goggles and gloves is necessary. Antiseptic mouth rinse is believed to reduce the viral load in the oral cavity; hand hygiene should be performed immediately after removing any kind of personal protective equipment. It must be ensured that proper cleaning and disinfection procedures are followed consistently in the examination room after each patient [7].

\section{Management guidelines for oral cavity squamous cell carcinoma}

Squamous cell carcinoma represents the most common form of head and neck cancer comprising approximately $90 \%$ of all head and neck malignancies [8]. Unlike the treatment of cancer arising from other anatomic sites of the head and neck, the primary treatment strategy for squamous cell carcinoma remains mainly surgical. Although multimodal therapy, including adjuvant radiation therapy with or without chemotherapy, is frequently used for advanced-stage disease, surgery represents the cornerstone in the management of this pathology [9]. During the COVID-19 pandemic, performing surgical procedures that expose health personnel should be avoided as much as possible. Therefore, the analysis of each particular case, relying on world protocols for the management of oral cancer, should lead to the correct management, including surgery only in those cases that are strictly necessary.
The TNM classification of malignant tumors is the most widely used system for cancer staging internationally. The system not only serves as a common language among providers of different specialties who collaborate in the multidisciplinary treatment of cancer patients but also acts as a guide to determine if the treatment should (or should not) be surgical [10]. The National Comprehensive Cancer Network recently updated the Clinical Practice Guidelines for cancers of the head and neck. The preferred treatment of squamous cell carcinoma remains surgery [11].

All early-stage and even most advanced-stage of carcinoma are amenable to surgical resection. For early-staged disease, surgery alone may be adequate initial treatment, sparing adjuvant radiotherapy with the associated long-term sequelae and morbidity. Nonetheless, for most patients with intermediate-staged to advanced-staged disease, adjuvant radiation alone or chemo radiation is indicated to reduce the risks of local and regional recurrence. The role of adjuvant therapy is based on the pathologic $\mathrm{T}$ staging and $\mathrm{N}$ staging after resection of the primary tumor and dissection of the neck lymph nodes at risk for metastasis [9]. Oral and maxillofacial surgeons must continually remain apprised of the most current staging algorithms and treatment pathways and adapt their clinical practice patterns to reflect contemporary standards of care relative to the management of squamous cell carcinoma.

\section{Procedures on patients with oral cancer: Clinical examination, surgeries, and urgencies}

During the peak of the COVID-19 pandemic, outpatient visits should be reduced to a minimum. Only urgent problems should prompt a patient to come to an outpatient unit or private practice. Among these urgent problems are patients who present oral cancer. Clinical examination must be carried out with personal protective equipment and should be limited to low risk cases or those patients where there is a low clinical suspicion of malignancy. Zimmermann, et al. [4] typified benign slowly growing tumors with an intermediate care priority, proposing the deferral of surgery for this type of cases until COVID-19 pandemic situation has settled as long as risks are under control. In the case of malignant disorders like oral cancer that need urgent surgical procedures, performing surgery in otherwise healthy patients is proposed. In the case of oncologic patients infected with COVID-19, surgery can be performed in the same way, reinforcing bio security measures and thorough monitoring during the recovery period.

Biopsies are indicated in the case of suspected malignancy due to the fact that this is considered an emergency procedure. Absorbable sutures should be used to minimize a second postoperative contact. Cleaning and disinfecting the exterior of the container after taking the sample should be performed as well [6]. Bleeding is a common oncologic emergency in patients with cancer [12]: In case of severe hemorrhage, considered as an emergency condition [4]. Appropriate surgery should be performed on healthy or infected patients. Situations where the growth of the tumor causes (imminent) airway compromise should also be managed surgically depending on the staging. 
The AO CMF International Task Force [13] also makes recommendations regarding the management of patients with oral cancer during the pandemic. If non-surgical methods (eg, radiation) can achieve similar results as a surgical approach, non-surgical therapy is recommended. However, surgical intervention is warranted in the following scenarios:

1. Cases in which a worse outcome is expected if surgery is delayed more than 6 weeks (squamous cell carcinoma of the oral cavity, oropharynx, larynx, hypopharynx)

2. Cancers with impending airway compromise

3. High grade or progressive salivary gland malignancy

4. T3/T4 melanoma

5. Salvage surgery for recurrent/persistent disease

6. High grade sino-nasal malignancy where non-surgical options will not be equally efficacious

All follow up appointments should be minimized in order to reduce patient-surgeon contact: 6-9 months for patients beyond the period of highest risk for recurrence (e.g. 18-24 months post-treatment). Patients in the immediate post-treatment phase must be prioritized and longer intervals between follow-ups as soon as suitable should be considered, even opting for telephone follow ups when possible and appropriate. Patients over 70 years of age (and/or with high risk co-morbidities, frailty) who fulfill urgent cancer criteria should be prioritized [14]. The guides for the provisioning of head and neck cancer services during the pandemic period are intended to guide and support the decisions of the surgeons.

\section{Recommended personal protective equipment for the attention of patients with Covid-19}

In the case of clinical examination, surgeons should be using a medical mask (FFP1 respirator/FFP2 respirator/N95 respirator/equivalent), a gown, gloves, cap and eye protection (goggles/face shield). Before taking a patient to the operating room, a test for SARS-CoV-2 should be performed. Any type of surgery performed on COVID-19 patients in the operating room should be practiced with FFP3 or N99 respirators, a disposable sterile gown, sterile gloves, a surgical hood cap and eye protection (goggles /face shield). Negative pressure must also be established to reduce the dissemination of the virus. The surgical team should not be present in the operating room during the process of intubation and extubation. At any time, the number of staff members in the operating room should be minimal. During the procedure, leaving or entering the operating room should be limited to a minimum. A time interval of 15 minutes must pass after the patient has left the operating room before the cleaning and disinfection process starts $[15,16]$.

\section{Role of virtual multidisciplinary team meetings}

Multidisciplinary team meetings that are attended by surgeons, medical oncologists, radiotherapists, radiologists, pathologists, and other specialists depending on the type of tumor to manage cancer patients provides the opportunity for patients to benefit from the experience of a larger number of professionals, especially when there is a complex or advanced case [17]. Currently, due to the risk of cancer patients being exposed to COVID-19, the role of a multidisciplinary team as an appropriate reference for deciding whether or not to undergo surgery and determining other treatment plans is crucial for the outcome. However, holding these meetings and bringing specialists (and patients, if necessary) together turns practically impossible due to hygienic conditions and social distancing.

In these particular circumstances, holding a virtual multidisciplinary team meeting via social media or video-conference is a more sensible way. One person as a coordinator can take history and all patient records, such as imaging and lab data, and share it to the rest. By examining the patient's condition and considering the current crisis, a treatment plan can be decided [5].

\section{Conclusions}

The influence of the COVID-19 pandemic on healthcare to the public is severe. The reduction of elective surgery is necessary; nevertheless, urgent oncologic and emergency surgery still has to be performed. Strategies must be developed to reduce the number of infections. The adequate approach of the COVID-19 challenge merits significant changes in the infrastructure of outpatient units, inpatient units, and operating rooms. Protecting patients as well as the medical staff is crucial. The care of the patient affected with oral cancer is imperative. Following strict bio security protocols, procedures such as clinical examinations for cancer patients and biopsies (to clarify diagnosis when malignancy is suspected) can be performed. In the case of benign slowly growing tumors, deferral of surgery until the COVID-19 pandemic situation has settled is recommended, as long as risks of deferral are under control. In the case of malignant tumors, surgery must be performed using appropriate biosecurity measures. Bleeding and tumors that interfere with the airway of the patient should also be approached surgically when possible. During the COVID-19 pandemic, performing surgical procedures that expose health personnel should be avoided as much as possible. Therefore, the analysis of each particular case, relying on world protocols for the management of oral cancer (like TNM classification of malignant tumors), should lead to the right management, including surgery only in those cases that are strictly necessary. These recommendations are based on the best, trustworthy and currently available information. As mentioned above, these are just recommendations and thus, the ultimate decision of the treatment of patients still relays with the health professional. The objective is to minimize the risk of infection, safeguarding what matters most: The lives of the patients.

\section{Disclosures}

All the authors declare that they have no conflict of interest. In addition, no funding was received for conducting this research.

\section{Acknowledgments}

None. 


\section{References}

1. Zhou P, Yang X-L, Wang X-G, et al. (2020) Discovery of a novel corona virus associated with the recent pneumonia outbreak in humans and its potential bat origin. bioRxiv 22: 914-952.

2. Toit $A D$ (2020) Outbreak of a novel corona virus. Nature Reviews Microbiology 18: 123.

3. Lu CW, Liu XF, Jia ZF (2020) 2019-nCoV transmission through the ocular surface must not be ignored. Lancet 395: e39.

4. Matthias Zimmermann, Emeka Nkenke (2020) Approaches to the management of patients in oral and maxillofacial surgery during COVID-19 pandemic. Journal of Craniomaxillofac Surg 48: 521-526.

5. Abolfazl Salari, Mohammad Shirkhoda (2020) COVID-19 pandemic $\&$ head and neck cancer patients management: The role of virtual multidisciplinary team meetings. Oral Oncol 105: 104693.

6. Mariana Villarroel-Dorrego (2020) Sars-cov-2 in the dental practice. Acta Odont. Venez. Edición Especial.

7. Kohn WG, Collins AS, Cleveland JL, et al. (2003) Guidelines for infection control in dental health-care settings. MMWR Recomm Rep 52: 1-61.

8. Marur S, Forastiere AA (2008) Head and neck cancer: changing epidemiology, diagnosis, and treatment. Mayo Clin Proc 83: 489501.

9. Ettinger K, Ganry L, Fernandes R (2019) Oral Cavity Cancer. Oral Maxillofac Surg Clin N Am 31: 13-29.
10. Amin MB (2017) American Joint Committee on Cancer. AJCC cancer staging manual. ( $8^{\text {th }}$ edn), New York: Springer.

11. National Comprehensive Cancer Network. Head and neck cancers (2018) NCCN clinical practice guidelines in oncology.

12. Gomes L, Stephen M, Jhingran A, et al. (2019) Short-course palliative radiation therapy leads to excellent bleeding control: $A$ single centre retrospective study. Clinical and Translational Radiation Oncology 14: 40-46.

13. Grant M, Schramm A, Buchbinder D, et al. (2020) AO CMF International Task Force Recommendations on Best Practices for Maxillofacial Procedures during COVID-19 Pandemic.

14. (2020) British Association of Head \& Neck Oncologists - Statement on COVID-19. BAHNO.

15. European Centre for Disease Prevention and Control (2020) Infection prevention and control for COVID-19 in healthcare settings.

16. World Health Organization (2020) Infection prevention and control during health care when COVID-19 is suspected: interim guidance. Geneva: World Health Organization.

17. El Saghir NS, Keating NL, Carlson RW, et al. (2014) Tumor boards: Optimizing the structure and improving efficiency of multidisciplinary management of patients with cancer worldwide. Am Soc Clin Oncol Educ Book 34: 461-466.

DOI: $10.36959 / 592 / 386$

Copyright: (c) 2020 Nicolás S, et al. This is an open-access article distributed under the terms of the Creative Commons Attribution License, which permits unrestricted use, distribution, and reproduction in any medium, provided the original author and source are credited. 